\title{
Yes, we are both cardiac surgeons: One family's unique perspective on work-life balance
}

\author{
Courtney J. Gemmato, MD, ${ }^{\mathrm{a}, \mathrm{c}}$ and Andrew C. W. Baldwin, MD ${ }^{\mathrm{b}, \mathrm{c}}$
}

My chosen career path was not intended to be that of a "trailblazer," nor was it designed to publicly challenge professional and social norms. Rather, my life-like many others-involved a series of difficult decisions, twists of fate, and inspirational mentors that led me to this point. With the tumult of surgical residency and fellowship now complete, I find myself better able to reflect on the unique nature of my life, job, and family, and have come to realize that our experience can serve as example-admittedly extreme-of the possibilities available to those contemplating a career in cardiac surgery (Video 1).

Despite increasing representation among surgical trainees, women in surgery are unavoidably aware of stereotypes and preconceptions that affect their day-to-day lives in the hospital. As my own career evolved, I unwittingly joined progressively smaller subsets of women for whom role models are increasingly rare. As a fullytrained cardiothoracic surgeon, I represent just $7 \%$ of the workforce reported by the Society of Thoracic Surgeons, and any hope of statistical context evaporates entirely when considering my cardiac focus, pregnancy during fellowship, and marriage to another cardiac surgeon-in-training.

Ours is thus a unique family experience within a field traditionally associated with outsized sex and work-life imbalances. Nearly every day, I find myself clarifying my job title to confused and skeptical onlookers:

\footnotetext{
From the ${ }^{\mathrm{a} D i v i s i o n}$ of Cardiac Surgery, Beth Israel Deaconess Medical Center; ${ }^{\mathrm{b}} \mathrm{Di}$ vision of Cardiac Surgery, Massachusetts General Hospital; and ${ }^{\mathrm{c} H a r v a r d ~ M e d i c a l}$ School, Boston, Mass.

Disclosures: The authors reported no conflicts of interest.

The Journal policy requires editors and reviewers to disclose conflicts of interest and to decline handling or reviewing manuscripts for which they may have a conflict of interest. The editors and reviewers of this article have no conflicts of interest.

Received for publication Aug 18, 2020; revisions received Sept 10, 2020; accepted for publication Sept 17, 2020; available ahead of print Nov 10, 2020.

Address for reprints: Andrew C. W. Baldwin, MD, Division of Cardiac Surgery, Massachusetts General Hospital, 55 Fruit St, Boston, MA 02114 (E-mail: Andrew, Baldwin@mgh.harvard.edu).

J Thorac Cardiovasc Surg 2022;163:179-83

$0022-5223 / \$ 36.00$

Copyright (C) 2020 by The American Association for Thoracic Surgery

https://doi.org/10.1016/j.jtcvs.2020.09.145
}

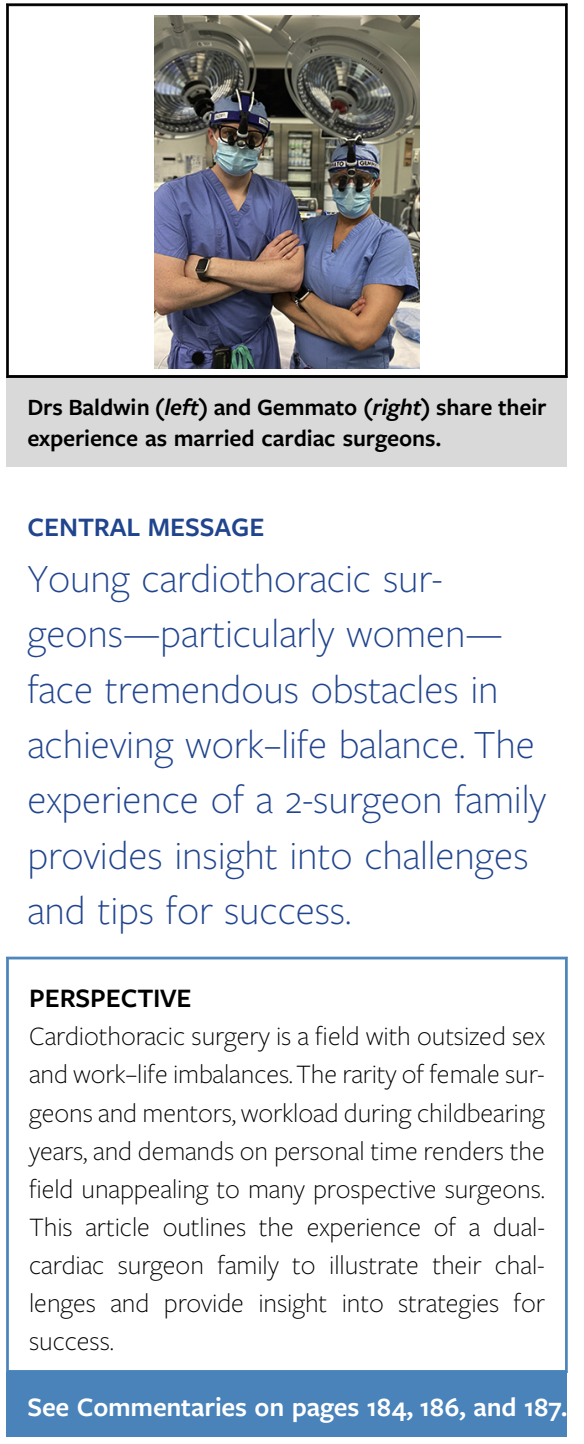

Yes, I really am a surgeon.

Yes, a real surgeon-just like my husband.

Yes, we are both cardiac surgeons.

While I understand that few will choose our path, I hope that our family's story can serve as a point of reference for young surgeons to better understand the possibilities-not just the limitations - of a life in surgery.

\section{Yes, It's Hard}

Women in medicine are all too aware of their minority status, and nearly all have a story that involves some form 


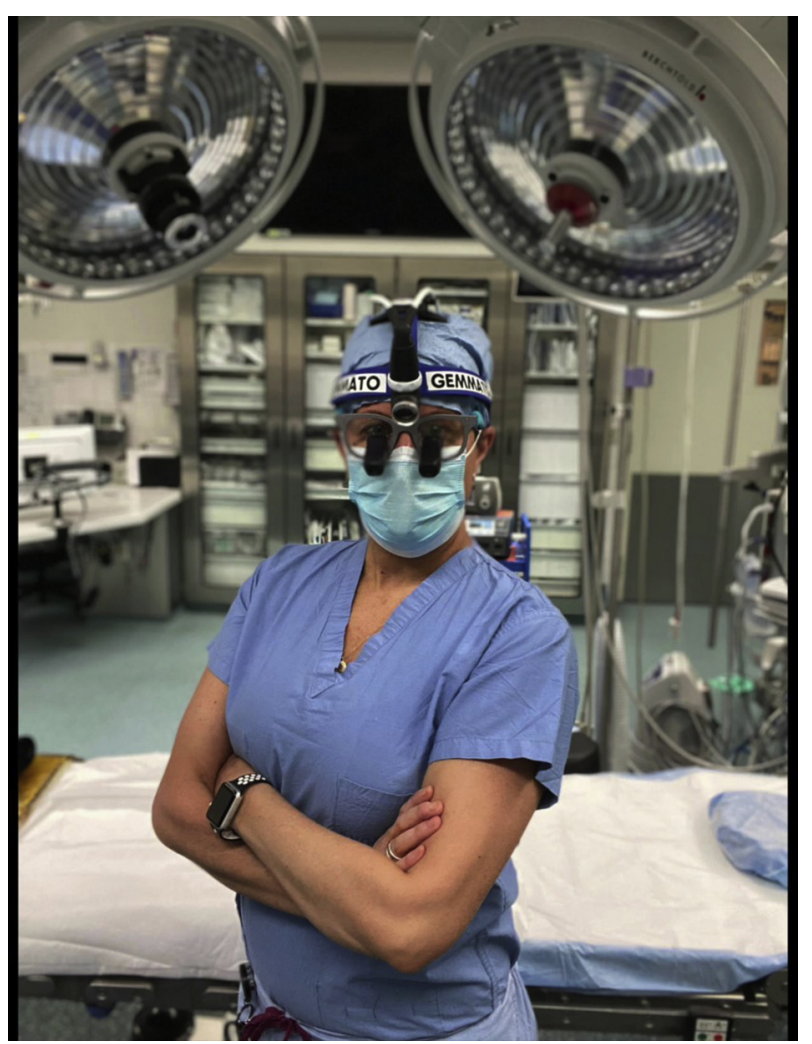

VIDEO 1. Drs Gemmato and Baldwin discuss the realities, challenges, and advantages of their lives as married cardiac surgeons. Video available at: https://www.jtcvs.org/article/S0022-5223(20)33042-7/fulltext.

of condescension, discrimination, or disbelief. Patients and doctors alike assume we aren't surgeons-in part because there are so few of us. While recent trends have shown increasing representation over time, less than one half of general surgery trainees are women - a percentage that falls to $20 \%$ among cardiothoracic surgery residents. ${ }^{2}$ Just over 200 women have ever been certified as diplomates of the American Board of Thoracic Surgery, and active female membership in the American Association for Thoracic Surgery constitutes less than $5 \%$ of that venerated society. ${ }^{3}$ Of the few women who complete cardiothoracic surgical training, only one third identify as cardiac surgeons - the remainder most often choosing careers in thoracic surgery or intensive care. ${ }^{4}$ The data simply reinforce what we all know to be true-female cardiac surgeons are rare.

One of the most commonly cited challenges facing women in cardiac surgery is childbearing. A recent survey of cardiothoracic surgeons found that women are not only more likely to delay having children than their male counterparts but also are more likely to believe their career will be adversely affected by family commitments. ${ }^{5}$ Additional studies have demonstrated increased pregnancyassociated complications, decreased fertility rates, and a fear of being perceived unfavorably among female physicians seeking children. ${ }^{6}$ Indeed, shouldering the physical and mental demands of pregnancy while maintaining an active cardiac surgical career is a daunting challenge. Lengthy operations, frequent emergencies, inconsistent meals, and primitive communal resident resources are particularly ill-suited to pregnancy and early motherhood. And while surgical programs are increasingly faced with adapting to the new reality of mothers-as-surgeons in the workplace, women physicians must also navigate traditional sex roles and expectations at home.

Dual-physician marriages have become increasingly common over the past several decades but remain statistically rare. In a recent study through the American College of Surgeons, approximately one half of surgeons reported a spouse working outside the home. Sixteen percent of those respondents were married to another physician, and just one third of that group (5\% of the working spouses) were fellow surgeons. ${ }^{7}$ A breakdown of surgical subspecialties participating in dual-surgeon households is not available, but available statistics and anecdotes would suggest that few involve 2 cardiac surgeons. Highlighting the domestic challenges for women in surgery, female surgeons are more likely to partner with a fellow doctor (36\% report a physician spouse compared with just $13 \%$ of men) and are much less likely to have a dedicated stay-at-home spouse ( $17 \%$ compared with $53 \%$ among male respondents). As is the case across the workforce, women surgeons thus enjoy less dedicated domestic support than their male counterparts while juggling the same clinical responsibilities. This discrepancy is exacerbated by the intense clinical, psychological, and temporal demands of a career in cardiac surgery.

Accommodating the schedule of one cardiac surgeon is a daunting challenge for any family. Managing a 2-cardiacsurgeon household is fraught with logistical challenges that can make each day feel like a shoestring catch. Even in the best of circumstances, the capricious nature of cardiac surgery means that no case can be relied upon as "straightforward." Emergencies are common and not infrequently result in sudden departures from home or extended periods in the hospital. Call schedules-often "in-house" for cardiac surgery trainees-can be burdensome and must be balanced between 2 spouses, frequently resulting in an alternating pattern that leaves one member of the household on-call at all times. Among all these occupational restrictions, childcare-from both an emotional and logistical perspective-is a considerable challenge. The majority of daycare facilities open around $7 \mathrm{AM}$ (when most surgeons have already rounded and are preparing for the start of their case) and close around 5 PM (before many second-start cardiac cases are finished). Employing full-time (60 hours/ week) nanny coverage is an expensive proposition for young surgeons and trainees who, on average, earn approximately $\$ 68,000$ per year. ${ }^{8}$ The worst-case scenario involving both parents responding to overnight emergencies 
further requires an overnight "backup" childcare option that is both costly and difficult to implement. In a 2-surgeon family, simply ensuring that an adult is present in the house can be a challenge. The guilt and frustration stemming from limited time with one's child is an additional stressor (aside from simultaneously managing the heart-lung machine) that is difficult to quantify.

\section{Yes, It's Possible}

The mere existence of my career serves as testimony to the opportunities available to any likeminded and determined women. I was raised in a small blue-collar town near Cleveland, Ohio, where a career as a heart surgeon was never even considered, let alone discouraged. My post-college resume serves as a stark reminder of the lack of strategic career counseling enjoyed by those from more affluent communities. Among other jobs, I worked as a receptionist for a feed mill, a telephone-based credit card collections agent, and a data entry staffer for a manufacturing plant before landing a job as a circulatory support technician that began my path towards a career in medicine. I was 30 years old when I started medical school and faced 11 more years of training. I am not supposed to be here.

Historical precedent would also suggest that I shouldn't have a child, but I do. In what was assuredly a statistical anomaly, 3 of the 4 cardiothoracic surgery fellows in my training program were women at the time of my arrivaleach giving birth at least once over the course of the fellowship. Our training program was admirably flexible and supportive of its gravid residents, and we found that the repetition-although challenging at times for coverage purposes-helped to normalize the concept of pregnancy within the residency, and we each benefitted from progressive institutional adaptations. While unusual, our experience signals a coming change in a fellowship process that has rarely entertained the possibility of a pregnant cardiac trainee (let alone 3).

For my part, I was well within the spectrum of advanced maternal age when my fellowship began and accepted the challenges of childrearing during my training to maximize our chances of starting a family. The calculus was not simple. I would be pregnant for 9 months during an intensive 2-year surgical fellowship and risk losing valuable operative experience. Revealingly, neither the Accreditation Council for Graduate Medical Education nor the certifying American Board of Thoracic Surgery mandates a universal parental leave policy, ${ }^{9}$ instead deferring to institution-specific policies that generally follow the guidelines of the Family and Medical Leave Act. My own maternity leave included all vacation time for the year and ultimately lasted 6 weeks, as any additional time off would have required an extension of my fellowship and risked separating our new family, as my husband was scheduled

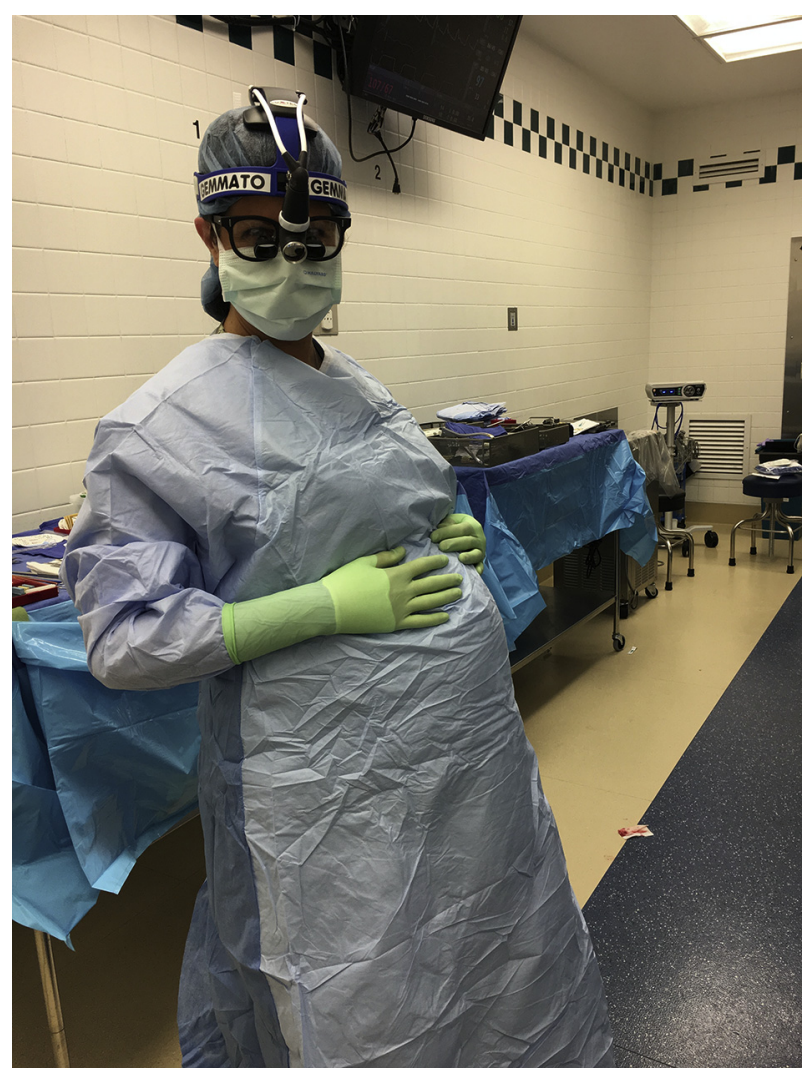

FIGURE 1. Dr Gemmato-then a cardiothoracic surgery fellow at Yale University-preparing for surgery while in her third trimester of pregnancy.

to start his own fellowship training immediately following my graduation. We lived on a resident salary and would likely need to hire full-time help upon my return to work. While my family and training program were incredibly supportive, the process was unsurprisingly fraught with challenges.

My preference was to be treated the same as any other nonpregnant trainee, but I soon found that physical limitations necessitated adaptations to my typical workflow. Throughout my pregnancy, I found that clear communication with the support staff, residents, and surgeons was critical for both my job performance and my health. Few attending surgeons are accustomed to their fellows taking bathroom or breast-pumping breaks during a coronary bypass operation or aortic aneurysm repair. Absences for illness or medical appointments and breaking scrub to use the restroom or stave off hypoglycemia would seem the most basic of allowances to the layperson but represent unusual departures from surgical custom. While occasionally awkward and undesirably personal, I found that clear communication of my needs during pregnancy was rarely (if ever) met with disapproval. In this way, I was able to operate until my 39th week of pregnancy by sacrificing a small 
amount of privacy (and expending a large amount of effort) (Figure 1).

As if our story weren't unusual enough, there is the small issue of my husband's career as a cardiac surgeon. We met before medical school and managed to survive the gauntlet of multiple residency matches, years of living apart, frantic weekend visits, and the uncertain compatibility of our future career paths (aside from the baseline stress of surgical residency). While we now have the good fortune of living in the same city, we face the daily challenge of raising a child amidst the operative schedules of 2 cardiac surgeons. Admittedly, we have endured the occasional crisis involving child hand-offs in the cardiac intensive care unit. But by and large, we have found a system that keeps our family afloat.

\section{Yes, It Takes Work}

Planning is essential. Our daily lives (and our sanity) depend on a carefully constructed scaffolding of schedules, communication, and contingencies. We have a full-time nanny who arrives at our house by $6 \mathrm{Am}$ and a grandparent who serves as an emergency backup. We coordinate our monthly call schedules in advance as much as possible. We avoid weekday calls on the same day but prioritize weekend calls together in an effort to maximize our time off as a family unit rather than staggering weekends and parenting separately. Backup options are confirmed in advance-for known overlaps in calls, expected long operative days, difficult handoffs, and even unexpected emergencies. Given its complexity, our monthly schedule is written on a large chalkboard in our kitchen to minimize confusion and provide clarity for all of our child's caretakers. The interwoven nature of our calls renders our schedule relatively inflexible. Clear communication-both asking for early schedule releases and communicating our inability to accommodate changes - has been critical to our success.

Cardiac surgery has a tendency to disrupt even the bestlaid plans. Cases run long, emergencies happen at inconvenient times, and the physicality and stress of the job can simply leave us exhausted. Maintaining perspective and perseverance through moments of adversity is a shared and important responsibility. Long distance, stress, selfdoubt, and frustration are common themes throughout surgical residency and fellowship. Added to that is the guilt stemming from rushed or lost time with our daughter as a result of our clinical obligations. We rely on our common understanding of the unique nature of our jobs to help support and buoy each other through the inevitable difficult times.

This perspective also helps us to remain patient with one another (within reason) when our resources and nerves are stretched thin. Several days of long cases can easily become a week without seeing our daughter. An unforeseen complication can derail a long-planned afternoon with the family. My husband and I rely on each other to weather these emotional storms and provide a level of understanding that can only be shared with a fellow surgeon. In this way, we have turned our unique common experience into an advantage rather than a burden. A constant work in progress, we have also learned the importance of compromise and equipoise between our individual careers. We share ideas, advice, and experience, and we expect to share patients in our future practice. Only by working togetherat home and on the job-can we find reasonable balance in our lives.

Our shared perspective also helps to strengthen another facet of our approach work-life balance - time management. Our profession is all-encompassing. Demands on our time are great, and it is tempting to use our time away from work to catch up, plan ahead, or re-examine clinical decision-making. Rather than risk distraction while at home with our daughter, my husband and I made a conscious decision to fully invest in our time together as a family. While at work, we are cardiac surgeons. While at home, we are parents. We will never abandon our obligations to our patients, but we actively work to protect our personal time. This approach can be difficult, as it requires cooperation and efficiency both at home and in the hospital, but it allows us to enjoy both aspects of our lives with fewer distractions. We are better doctors when we are happier at home.

\section{Yes, the Culture Must Change}

Surgical culture is rooted in tradition. Prototypical cardiac surgeons prioritized their job above all else, and expected their families to admire, adapt, and accommodate. Historically, programs focused little on the individual needs of their surgeons and trainees, instead regarding personal requests as a sign of weakness or lack of dedication. Cardiothoracic surgeons have long believed that training should involve a form of absolute dedication and self-sacrifice capable of producing a steel-hardened finished product. However, the landscape of medicine is changing, and we must not be content with traditional paradigms that discourage future generations of surgeons from joining our ranks.

Cardiac surgical training will always be difficult and require tremendous sacrifice. It should. The responsibilities are great, and the consequences are high. But if we are to attract the most talented candidates and expect them perform at their best, we cannot continue to ignore the great personal costs. Medical students and surgical residents should not have to query PubMed for articles (like this) to gauge if it is even possible to have a family and a career. The culture of surgery should move to embrace those with families rather than view them with skepticism regarding their commitment or reliability. Small changes can have a great impact. 
Time is a precious commodity for cardiac surgeons, particularly trainees. Establishing a culture that respects the value and importance of personal time is critical for the recruitment, retention, and wellbeing of future generations of cardiac surgeons. Time outside the hospital should be protected from burdensome levels of home call, administrative work, and off-hours meetings. Improved and flexible coverage options (such as physician extenders) should be viewed as a necessary investment in the wellness of our surgeons, not as an expensive luxury. By nature, cardiac surgery will never be a 9-to-5 job, but to suggest that the alternative must be $24 / 7$ is a false choice. The value of my fellowship training is not diminished by acknowledging that many of my responsibilities were more valuable to the institution than my own education. Likewise, I am capable of being a dedicated and conscientious cardiac surgical attending while periodically entrusting the care of my patients to colleagues. We must do more to protect ourselves and our families from the false belief that the job must be all-encompassing.

Regardless of how we safeguard our personal time, the dedication required of a cardiac surgeon is a strain on any family. Surgical performance and fast-paced clinical decision-making are exponentially more difficult when clouded by uncertainty about childcare plans at home. Rather than ignoring domestic obligations with a level of plausible deniability, programs should seek to support and nurture family-oriented surgeons through programs such as extended hours' childcare coverage and protected longterm family and postpartum leave. Investments in the lives of our surgeons will pay dividends through improved physician wellbeing, enhanced clinical care, and successful recruitment of future generations. While the factors involved in physician burnout are multivariate, proactive measures to support surgeons with families would help convey a sense of value and allow for improved wellness practices amongst the workforce.

\section{Yes, More Challenges Await Us}

We are proud to have emerged from the crucible of training with our family intact (and largely unscathed), but we understand that many more obstacles await us. With my husband's graduation from fellowship, we now face a unique job search involving 2 junior surgeons in search of highly specialized jobs in close proximity. Hospitals in the same region pose the challenge of distance and conflicting interests, while jobs in the same practice can result in alternating call schedules and unwanted domestic strife in the operating room. Physician couples further hold a distinct disadvantage in contract negotiations, as geographic restrictions (to keep families intact) increase the risk of undercompensation and limit opportunities for career advancement. The more senior surgeon, I have already fielded lower-value contracts than those offered to my less-experienced spouse-a phenomenon of professional and societal inequity that extends throughout the workforce. ${ }^{10}$ Childcare is an ever-present logistical problem for our family regardless of future earning potential. We will always face the challenge of balancing our working and domestic lives, especially as our careers become more intertwined.

Despite these hurdles, we have found many advantages to our dual-surgeon status. Our shared experience and common appreciation for the stresses in our profession allows for a deeper level of understanding and support. When faced with the heartbreak of missing a family event or longawaited time with our daughter, we support each other with a level of encouragement and acceptance that would be difficult to expect of someone outside the field. We have round-the-clock access to specialized clinical expertise, and never have to feel alone in our decision-making or selfconscious about asking a colleague for advice. When faced with disappointing outcomes, frustrations, or long and physical days that are inevitable in this profession, we find comfort in having a support system that really does "get it."

Ours is an extreme example, but our careers have not stopped us from pursuing a full and complete life. Those with aspirations of a career in cardiac surgery should take solace in knowing that pregnancy, family, marriage, and mutually supportive careers are indeed possible. Many of the challenges we face are inseparable from the job, but there is much we can do as a field to better assist and empower female and family-oriented surgeons. While few will follow our family's unusual path, we hope that those who make similar choices will be supported and embraced as a new generation of \#surgeonfamilies.

\section{References}

1. Ikonomidis JS. The Society of Thoracic Surgeons Thoracic Surgery practice and access task force: 2014 workforce report. Ann Thorac Surg. 2016;102:2118-26.

2. Stephens EH, Robich MP, Walters DM, DeNino WF, Aftab M Tchantchaleishvili V, et al. Gender and cardiothoracic surgery training: specialty interests, satisfaction, and career pathways. Ann Thorac Surg. 2016;102:200-6.

3. Antonoff MB, David EA, Donington JS, Colson YL, Litle VR, Lawton JS, et al. Women in thoracic surgery: 30 years of history. Ann Thorac Surg. 2016;101: 399-409.

4. Donington JS, Litle VR, Sesti J, Colson YL. The WTS report on the current status of women in cardiothoracic surgery. Ann Thorac Surg. 2012;94:452-9.

5. Pham DT, Stephens EH, Antonoff MB, Colson YL, Dildy GA, Gaur P, et al. Birth trends and factors affecting childbearing among thoracic surgeons. Ann Thorac Surg. 2014;98:890-5

6. Klebanoff MA, Shiono PH, Rhoads GG. Outcomes of pregnancy in a national sample of resident physicians. N Engl J Med. 1990;323:1040-5.

7. Dyrbye LN, Shanafelt TD, Balch CM, Satele D, Freischlag J. Physicians married or partnered to physicians: a comparative study in the American College of Surgeons. J Am Coll Surg. 2010;211:663-71.

8. Martin KL. Medscape residents salary \& debt report: 2019. Available at: https://www. medscape.com/slideshow/2019-residents-salary-debt-report-6011735. Accessed June 16, 2020.

9. American Board of Thoracic Surgery: related policies statement. Available at: https://www.abts.org/ABTS/CertificationWebPages/Related\%20Policies.aspx. Accessed June 16, 2020.

10. Freund KM, Raj A, Kaplan SE, Terrin N, Breeze JL, Urech TH, et al. Inequities in academic compensation by gender: a follow-up to the national faculty survey cohort study. Acad Med. 2016;91:1068-73. 